\title{
Study on the Method of Angle Domain Analysis and Active Compensation for Roll Eccentricity
}

\author{
Tao Liu
}

Hebei Provincial Key Laboratory of Heavy Machinery

Fluid Power Transmission and Control

(Yanshan University)

Qinhuangdao, 066004, China

liutao@ysu.edu.cn

\author{
Yan Dan \\ Environmental Management College of China \\ Qinhuangdao, 066004, China \\ yandan8463@163.com
}

\begin{abstract}
This article carries out a new method to find out and compensate the roll eccentricity online in the strip cold rolling process. Firstly, the roll eccentricity signals are found out from the massive process data by a soft measurement method, secondly, this signals in time domain is converted into signal in angle domain with strict periodicity. Then the values of roll eccentricity in every sampled angle of one cycle are saved in a special array, and added inverse to the setting value of the position loop of the AGC system, adjusting the roll gap \& compensate the roll eccentricity activity. Finally, the rolling experiments prove that this method is accurate and sTable, and the roll eccentricity disturbance is inhibited obviously.
\end{abstract}

Keywords- Cold Strip rolling; Roll Eccentricity; Angle Domain Analysis; Active Compensation

\section{INTRODUCTION}

In the strip rolling process, roll eccentricity of strip mill affect thickness control precision of hydraulic AGC (automatic gauge control) system directly. Limited by field conditions, the roll eccentricity are often difficult to detect online. Therefore, the analys is method of roll eccentricity is important to compensate roll eccentricity in hydraulic AGC system ${ }^{[1]}$.

Actually, the rotating speed of roll will change now and then in the rolling process. Although the roll eccentricity signal fluctuates cyclically to some extent, it should belong to the non stationary signal essentially in time domain. If the measured eccentricity signal is used for active compensation directly, there may be frequency deviation and phase deviation between compensating value and actual roll eccentricity, and the effect of eccentricity compensation will be limited ${ }^{[2]}$. At the same time, the roll eccentricity signal is comprised of the eccentricity of support rollers and work rolls. When the slip occurs between the work rollers and support rollers, the eccentricity signal will change ${ }^{[3]}$. The roll eccentricity compensation may be wrong, and the stability of rolling and the accuracy of thickness may get worse seriously.

According to order analys is principle, the time do main signal of rotating machinery can converse into angle domain signal ${ }^{[4-5]}$. The conversed roll eccentricity signal in angle domain is periodic strictly, and the characteristic is easy to be extracted and saved. Using the roll eccentricity analysis method online, the data of eccentricity characteristics are got out and saved periodically, and the current roll eccentricity signal is predicted based those saved date in angle domain.

\section{PRINCIPLE OF ROLL ECCENTRICITY COMPENSATION IN ANGLE DOMAIN}

The roll eccentricity is geometric features of all the rollers, including working rollers and supporting rollers. Because the diameter of supporting rollers is far greater than that of working rollers, the cycle of roll eccentricity equals the rotating cycle of supporting rollers basically.

Mark the $\mathrm{N}$ as the number of the discrete points in a cycle, the roll eccentricity signal could be sampled and saved as array. Here $\mathrm{n}$ is the number of angle sequence. $\mathrm{n}=1,2,3 \ldots N$. Selecting a zero crossing point as the begin point, the discrete characteristic data of the roll eccentricity is shown in Fig. 1.

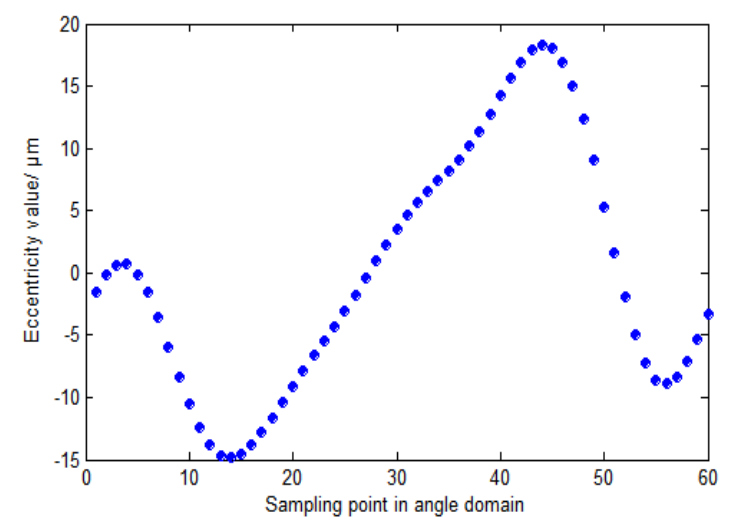

Figure 1. Characteristic data of the roll eccentricity in one angle domain cycle

Auto gauge control (AGC) system is a control system in time domain. As an additional subsystem of AGC, the REAC (roll eccentricity analysis and compensation) system must exchange data in time domain. As shown in Fig. 2, the REAC system comprise some function modules, such as the analysis in time domain, the conversation from the time domain to the angle domain, the data saving and updating, the eccentricity signal prediction, the phase calibration and so on. All those are linked together to a whole system. 


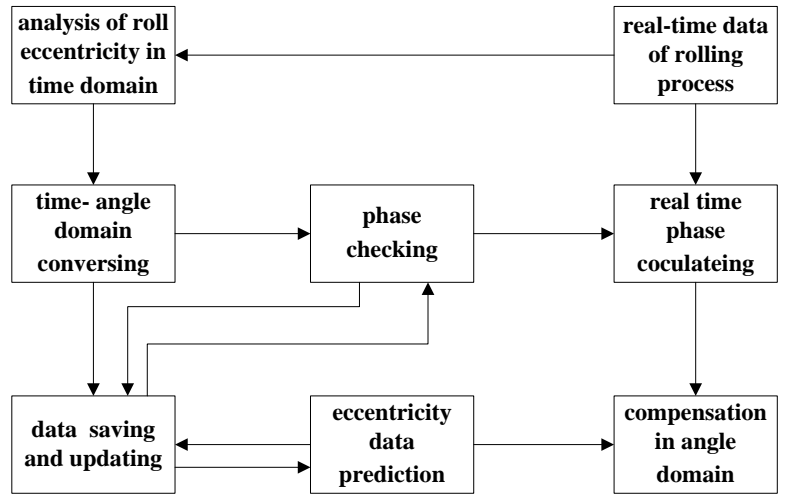

Figure 2. Principle of roll eccentricity compensation in angle domain

\section{THE ANALYSIS OF ROLL ECCENTRICITY}

A. The analysis of roll eccentricity in time domain

The mill spring Equation is described as:

$h=S_{0}+\frac{P}{M}$

$h$ _ the strip exit thickness, mm;

$S_{0}$

$P$ - no-load roll gap, $\mathrm{mm}$

$P$ _ the press force, $\mathrm{KN}$;

$M$ the longitudinal stiffness of rolling mill, $\mathrm{KN} / \mathrm{mm}$;

The no-load roll gap $S_{0}$ contains structure constant of rolling mill, the displacement of servo hydraulic cylinder $X_{P}$, and the changes of roller size in radial direction, including thermal expansion of rollers $X_{t}$, the roll eccentricity ${ }_{\mathrm{e}}$, and the wear of rollers ${ }_{m}$. Ignoring the wear and the thermal expansion of rollers, which changes slowly in a long time, and using differential form, the soft measurement model of roll eccentricity will be:

$$
\Delta x_{e}=\frac{\Delta P}{M}-\Delta h-\Delta x_{p}
$$

$\Delta X_{P}$ the displacement of servo hydraulic cylinder in differential form, mm;

$\Delta X_{e}$ the roll eccentricity in differential form, $\mathrm{mm}$;

Using this model, the time domain roll eccentricity data are calculated from real-time rolling data. They can be described as a discrete array $x_{\mathrm{e}}\left(t_{\mathrm{i}}\right), t_{\mathrm{i}}$ are the sampling times, $i=1,2,3 \ldots n$.

\section{B. The time- angle domain conversing method}

The roll eccentricity in time domain can be transformed to angle domain using resample method [6-7].

Supposing the resample times in same angle space are $T_{\mathrm{n}}$ $(n=1,2,3 \cdots N$ ), the instantaneous velocity is $v(t)$, those resample times $T_{n}$ can be calculated from the following integral:

$$
\begin{aligned}
& 2 \pi \int_{\mathrm{T}_{0}}^{T_{n}} v(t) \cdot \mathrm{dt}=n \Delta \theta \\
& \mathrm{n} \longrightarrow \text { the number of angle unit; } \\
& \Delta \theta \quad \Delta \theta=2 \pi / N ;
\end{aligned}
$$

Solving this integral Equation, the effective solutions $T_{\mathrm{n}}$ are resample times in equal rotating angular. The roll eccentricity values at those moments $T_{n}$ could be computed by the Langrange linear interpolation formula:

$$
\begin{aligned}
& x_{n}=x_{e}\left(\mathrm{t}_{\mathrm{i}}\right)+\frac{x_{e}\left(t_{\mathrm{i}+1}\right)-x_{e}\left(t_{\mathrm{i}}\right)}{t_{i+1}-t_{i}}\left(T_{n}-t_{i}\right) \\
& t_{i} \leq T_{\mathrm{n}} \leq t_{i+1}
\end{aligned}
$$

\section{The time- angle domain conversing method}

In order to eliminate the accumulative phase error brought with the time-angle domain conversation, the phase of eccentricity cycle needs to be checked. For this purpose, the correlation function between the last eccentricity data and that stored eccentricity data of the cycle before the last one should be calculated with Eq.(5)[8]:

$$
R(\tau)=\frac{1}{\mathrm{n}} \sum_{\mathrm{i}=1}^{60} x_{i} y_{i+\tau} \quad \tau=-1,0,1
$$

$X_{\mathrm{i}} \_$last eccentricity data

$y_{\mathrm{i}} \_$stored eccentricity data of the cycle before the last one;

Because eccentricity signals is periodic, in Eq.(5):

$$
y_{i+60}=\mathrm{y}_{\mathrm{i}}
$$

If the $R(0)$ is the maximu m, the phase of last eccentric data is accurate, then save the data directly;

$$
y_{i}=x_{\mathrm{i}}
$$

if $R(1)$ is the maximum, the phase of last eccentric data lag a angle domain unit, then the data should be moved forward sequentially before being saved;

$$
y_{i+1}=x_{\mathrm{i}}
$$

if $R(-1)$ is the maximu $\mathrm{m}$, the phase of last eccentric data lead a angle domain unit, then the data should be moved backward sequentially before being saved.

$$
y_{i-1}=x_{\mathrm{i}}
$$

\section{Roll eccentricity prediction}

The roll eccentricity signal is comprised of the eccentricity of working rollers and that of supporting rollers, when the slip occurs between the work roll and supporting roll, subtle changes will occur in the eccentricity signal. Even so, the roll eccentricity signal will still maintain a sTable trend. Therefore, it is possible to predicate the eccentricity data occurs in the next 
compensating cycle based on those eccentricity data in last several cycles. According to the exponential smoothing prediction method, the predicated eccentricity values are calculated by next formula ${ }^{[9]}$.

$$
\hat{y}_{t+m}=\left(2 S_{t}^{(1)}-S_{t}^{(2)}\right)+\frac{m \alpha}{1-\alpha}\left(S_{t}^{(1)}-S_{t}^{(2)}\right)
$$

$$
S_{t}^{(2)}
$$

the two index exponential smoothing prediction values at cycle $\mathrm{t}$, it can be calculated by Equation (11).

$$
S_{t}^{(1)} \text { the one index exponential smoothing }
$$
prediction values in cycle $t$. it can be calculated by Equation (12).

$$
\begin{aligned}
& \alpha \text { the weights of measured values } \\
& m \text { the number of eccentricity cycles to predicate } \\
& S_{t}^{(2)}=\alpha S_{t}^{(1)}+(1-\alpha) S_{t-1}{ }^{(2)}
\end{aligned}
$$

$$
S_{t}^{(1)}=\alpha y_{t}+\left(\begin{array}{ll}
1 & \alpha
\end{array}\right) S_{t}{ }^{(1)}
$$

$$
y_{t} \text { _the measured values in cycle } \mathrm{t}
$$

The template is used to format your paper and style the text. All margins, column widths, line spaces, and text fonts are prescribed; please do not alter them. You may note peculiarities. For example, the head margin in this template measures proportionately more than is customary. This measurement and others are deliberate, using specifications that anticipate your paper as one part of the entire proceedings, and not as an independent document. Please do not revise any of the current designations.

\section{COMPENSATION OF ROLL ECCENTRICITY}

Because the thickness meter for outlet plate must be installed in the position $\mathrm{L}$ distance behind roll gap, the thickness signals lag $\mathrm{K} \cdot \Delta \theta$ angle, it can be calculated as follows:

$$
K=\left|\frac{L}{(1+S) \Delta \theta}\right|
$$

\section{$S$ _ Forward slip coefficient}

Therefore the last eccentricity signal according to Eq.(2) lag $\mathrm{K}$ angle domain unit to the real eccentricity signal at the same time. If the last phase of soft measured eccentricity signal is $\mathrm{M}$, the compensation value $E_{O}$ should be Look up from array $\hat{y}_{n}$ according the Serial number $\mathrm{M}+\mathrm{K}$, and send to AGC system.

$$
E O=\hat{\mathrm{y}}_{\mathrm{M}+\mathrm{K}}
$$

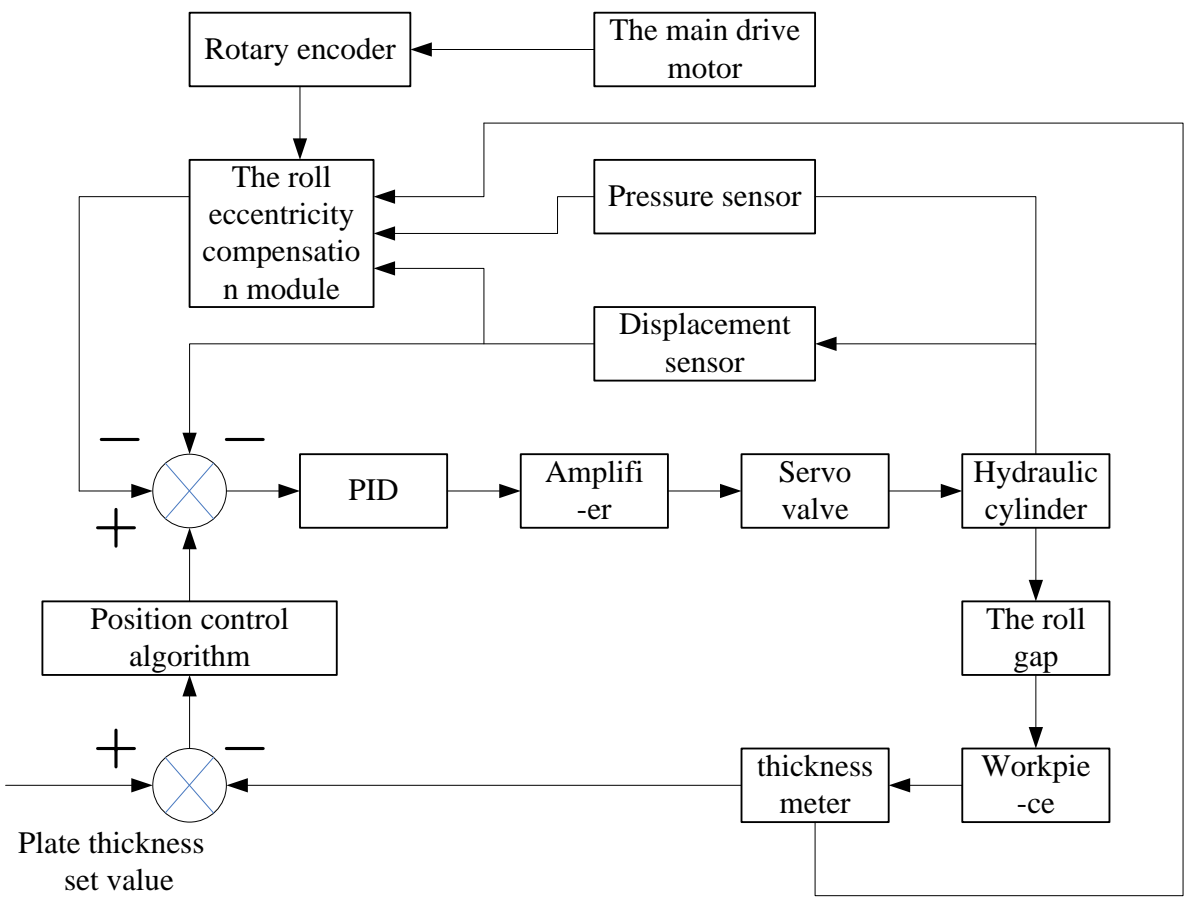

Figure 3. AGC system with the roll eccentricity compensation 
The AGC control system with the roll eccentricity compensation is shown in Fig. 3. The AGC system includes the thickness outer loop and the hydraulic cylinder position inner loop. In the inner loop, the setting value of position is calculated by position control algorithm in the outer loop, subtracting the feedback signal in comparers, then sent into the PID module, the amplifier, and the servo valve. Where the electric signal is amplified and transformed in hydraulic flow to the hydraulic cylinders. The displacement of cylinders is measured by sensors, and the signal of sensors is feedback to the comparers. In the outer loop, the setting values of thickness is given by upper computer, is subtracted the feedback thickness signal in comparers, and the deviation value is sent to the position control algorithm, to calculate the setting value of cylinder position.

Based on the traditional AGC system, the REAC module is added to eliminate the thickness fluctuation caused by roll Eccentricity. In this module, the signals from sensors in mill are used to analyze the roll eccentricity online. Then the roll eccentricity in time domain is transform into angle domain, and saved and phase checked according the algorithm above. Then the prediction of roll eccentricity is output and added inverse to the setting value of position, adjusting the roll gap \& compensating the roll eccentricity activity. ${ }^{[10]}$

\section{ROLLINGEXPERIMENT WITHROLL ECCENTRICITY COMPENSATION}

The rolling experiments were carried out in $300 \mathrm{~mm}$ four roller reversible cold rolling mill. The operating parameters of the experiment are shown in Table 1.

TABLE I.

EXPERIMENTAL PARAMETERS

\begin{tabular}{c|c|c}
\hline Parameter & Unit & Value \\
\hline Radius of working rollers & $\mathrm{mm}$ & 45 \\
\hline Radius of supporting rollers & $\mathrm{mm}$ & 300 \\
\hline Width of roller & $\mathrm{mm}$ & 210 \\
\hline Width of strip & $\mathrm{mm}$ & 0.5 \\
\hline Thickness of strip & $\mathrm{mm}$ & $08 \mathrm{Al}$ \\
\hline Material of strip & & 5000 \\
\hline Tension & $\mathrm{N}$ & 1.5 \\
\hline The positive bending force & $\mathrm{T}$ & 0.5 \\
\hline The rolling speed & $\mathrm{m} / \mathrm{s}$ & 36 \\
\hline The rolling force & $\mathrm{T}$ & ( \\
\hline Using the same & experimental & parameters and
\end{tabular}

Using the same experimental parameters and condition, two group rolling process and their output thickness of trip are compared in Fig. 4. One is the rolling process without the REAC module, and the curve of thickness error is showed in Fig. 4(a), the other one is the rolling process with the REAC module showed in Fig. 3, and the curve of thickness in differential form is showed in Fig. 4(b). It is proved by experiments that the largest thickness errors could be reduced from to , and the cyclical fluctuation in the thickness curve could decrease obviously.

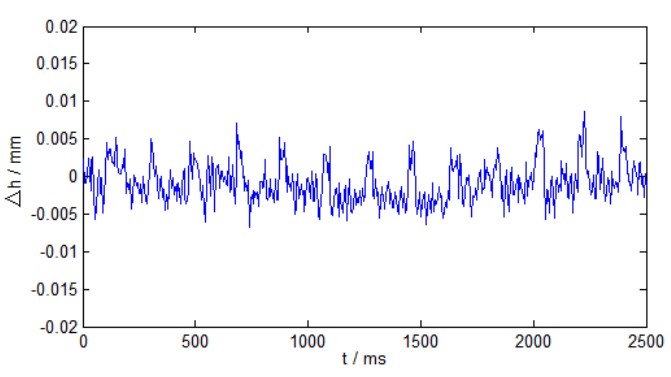

(a)

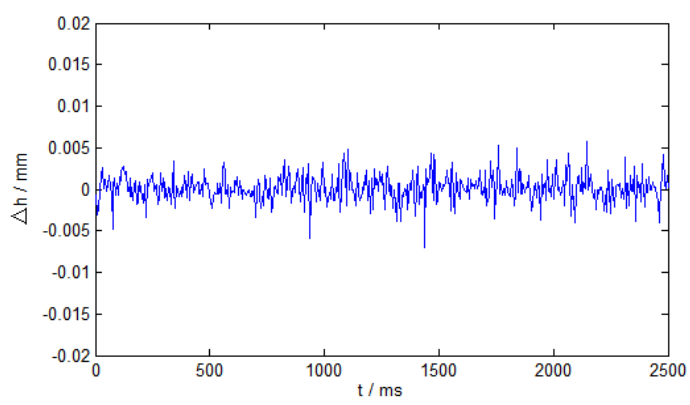

(b)

Figure 4. thickness curve comparing

\section{CONCLUSIONS}

(1) By presenting a roll eccentricity angle domain analys is method, the converted roll eccentricity signal in angle domain has a strictly periodic, and is easy to be feature extraction and preservation.

(2) By feature data updating and phase checking, this compensation method can adapt the change of the roll eccentricity signal. It improves the accuracy and adaptability of eccentricity compensation.

(3) It is proved by the rolling experiments that the roll eccentricity in angle domain analysis and compensation method can reduce the maximum thickness errors, and eliminate thickness cyclical fluctuation obviously.

\section{ACKNOWLEDGMENT}

This paper supported by the Province Natural Science Foundation of Hebei (No. E2013203126) and Native Natural Science Foundation of China (Grant No. 51305389).

\section{REFERENCES}

[1] NIU Manke, YANG Weidong, ZHANG Lijuan. Phase domain filtering pick up method for roll eccentricity compensation control[J]. Journa 1 of University of Science and Technology Be ijing. 2011,33(1):99-104

[2] CHEN Zhiming, LUO Fei, XU Yuge, YU Wei. Roll Eccentricity Compensation Based on Anti-Aliasing Wavelet Analysis Method [J]. Journal of Iron and Steel Research, International. 2009, 16(2): 35-39

[3] Li Ning, Qin Shuren, Mao Yongfang, et al. Gabor Order Tracking Filtering Technology in Rotary Machinery. Chinese Journal of Mechanical Engneering 2010,23(5): 613-619

[4] Liu Jingfang, Yu Yueqing, Huang Zhen, et al. General Order Principle for Multi-Bennett Linkages. Chinese Journal of Mechanical Engneering. Chinese Journal of Mechanical Engneering 2013,26(2): 275-281 
[5] Peng Fuqiang, Yu Dejie, Wu Chunyan. Self-adaptively Time varying Filter Based Order Tracking Method and ItsApplication in Gearbox Fault Diagnosis. Journal of Mechanical Engneering. 2012, 48(7): 77-85

[6] Zhao Xiaoping Hou Rongtao. Gabor Order Tracking Based on Viterbi Algorithm. Journal of Mechanical Engneering. 2009, 45(11): 247-252

[7] Chen Zhifei, Sun Jincai, Niu Yilong. A Better Method of Frequency Estimation for Sinusoidal Signals Using Coherent Average [J]. Journal of Northwestern Polytechnical University. 2009, 27(3): 387-390
[8] Christopher T A, Shadab N M, Torry L A. Suppressing Coherent Noise in Radar Applications with Long Dwell Times. IEEE Geo science and Remote Sensing Letters, 2005, 2( 3) : $284 \sim 286$

[9] LI Suo-ping, LIU Kun-hui.Quadric Exponential Smoothing Model with Adapted Parameter and Its Applicat ions[J].Systems Engineering-theory \& Practice.2004,(2):95-99. (in Chinese).

[10] Li Boqun, SUN Yikang, Fu Jian, et al. Control of Roller Eccentricity Based on Phasic Compensation Strategy[J]. Journal of Iron and Steel Research. 2006, 18(6): 55-58(in Chinese) 